\title{
Structural Evaluation for Distribution Networks with Distributed Generation Based on Complex Network
}

\author{
Fei Xue, ${ }^{1}$ Yingyu Xu, ${ }^{1}$ Huaiying $\mathrm{Zhu}^{2}$ Shaofeng Lu, ${ }^{1}$ Tao Huang, ${ }^{3}$ and Jinling Zhang ${ }^{1}$ \\ ${ }^{1}$ Department of Electrical and Electronic Engineering, Xian Jiaotong-Liverpool University, No. 111 Ren'ai Road, \\ Suzhou Industrial Park, Suzhou, China \\ ${ }^{2}$ SDIC Baiyin Wind Power Co., Ltd., Lanzhou, China \\ ${ }^{3}$ Politecnico di Torino, Torino, Italy \\ Correspondence should be addressed to Fei Xue; fei.xue@xjtlu.edu.cn
}

Received 16 May 2017; Revised 1 September 2017; Accepted 12 September 2017; Published 17 October 2017

Academic Editor: Zofia Lukszo

Copyright (C) 2017 Fei Xue et al. This is an open access article distributed under the Creative Commons Attribution License, which permits unrestricted use, distribution, and reproduction in any medium, provided the original work is properly cited.

\begin{abstract}
Structural analysis based on complex network theory has been considered promising for security issues of power grids. At the same time, modern power distribution networks with more Distributed Generations (DGs) and Energy Storage Systems (ESS) have taken on more challenges in operation and security issues. This paper proposed a dedicated metric named as Power-SupplyAbility for power distribution networks based on net-ability. Special features of DGs, such as relations of capacities, identification of effective supply area, and limitation in continuous power supply, have been considered in definition. Furthermore, a novel opinion is proposed that the extent of improvement for operation and security by adding DGs also depends on the original structure of the distribution networks. This is an inherent ability of the original networks and could be quantitatively analyzed. Through case studies, this method has been proved to be effective in identifying potential structural vulnerabilities of distribution networks; particularly the impact of DGs on security has been studied. Furthermore, it can help in site selection for DGs by providing different priorities of locations compared with results of other works. This can help to complement other methods to construct a more comprehensive methodology by considering aspects of security, economy, and quality.
\end{abstract}

\section{Introduction}

The networks of power system, also named as power grids, are a critical infrastructure in modern social livings [1]. Serious outages of electrical power systems can impact the whole society $[2,3]$. For instance, recent blackouts have occurred in the USA and Europe directly causing losses up to billions of dollars [4]. These serious consequences have drawn much attention to electrical security problems such as accidental or intentional attacks $[4,5]$.

However, with the increasing size and complexity in power grids [6], as well as increased consumption of power and other social developments, it is becoming more difficult and complex to analyze a large scale of whole power system and the complicated interconnectivity precludes us from understanding and evaluating an overall power system [6]. Complex network theory is a popular method to analyze networked systems in terms of a set of lines with connected nodes and fortunately recent studies have found that several properties of complex network $(\mathrm{CN})$, such as small-world [7] and the characterization of scale-free [8], were related to power networks. It then allows structural analysis of power grids through pure topological metrics and brings a new analyzing method to overcome the above problems $[9,10]$. In the theory of $\mathrm{CN}$, Global Efficiency is a metric to measure the efficiency of the network for information transmission between vertices [10] and later is popularly referred to in assessing the vulnerability of power grids [5, 11-15]. Furthermore, with consideration of specific features in electrical engineering, it was updated as net-ability to evaluate power transmission networks $[2,4]$. Based on complex network concept, net-ability analyzes the performance of transmission networks through considering extra physical features such as 
power flow limits, electrical distance, and contributions of all involved transmission paths.

Although net-ability is effective in analyzing power transmission networks, due to different structural features among transmission and modern distribution networks, it could not be directly utilized for modern distribution networks. In conventional distribution networks, the main differences from transmission networks are their radial topology and impedance to resistance ratio. But it is not a problem for complex network theory to analyze any network structure including radial topology, and it is not a problem to adapt net-ability with appropriate impedance model. However, in modern power distribution networks, Distributed Generation (DG) and energy storage devices may increase its complexity. DGs, simply defined as small-scale electricity generation within distribution networks or on the customer side of the networks, are currently undergoing an increasing amount to relieve environmental problems, such as greenhouse gases from traditional electricity generation [16-23]. There are various categories of DGs, such as photovoltaic cells installed at homes or wind turbines on a farm land. Although these DGs could improve power supply to local loads, compared to conventional generation, they are highly dependent on climates or weather and could not provide continuous electricity $[16,17]$. So battery Energy Storage Systems (BESS) can also be widely applied to improve power stability and demand/supply balance [17].

Up to now, to our best knowledge, few papers have considered direct applying of complex network approaches for analyzing modern distribution networks, especially to include impacts from DG and BESS. By comparing with conventional power distribution stations, [24, 25] consider DG with relevant small capacity and being placed to load node. Reference [24] pointed out that the power supply performance of DG should decrease rapidly with the increases of distance. Based on complex network concept, they raise some metrics to reflect that DG performance would decrease rapidly with increase of distance. The metrics used exponential form to show that DG contributed more power to relatively local load demand and less to remote loads, but exponential form cannot be fully justified and specific electrical features, such as impedance, power capacities of different devices, and contribution of different paths, were not fully considered. In this paper, the main impacts from DG on structural analysis of distribution networks are considered according to the channel capacity, supplying distance as well as intermittency and fluctuation of primary energy. These will be discussed in detail in Sections 2 and 3.

The rest of paper is organized as follows: global efficiency and net-ability will be discussed with their limitations for applying in distribution networks in Section 2. In Section 3, a new concept of Power-Supply-Ability (PSA) and its application in evaluating power distribution networks will be proposed. In Section 4, a novel opinion will be discussed to evaluate the inherent ability of original network structure to be improved by adding DGs. Simulation and results of application of PSA are shown in Section 5 with some examples and Section 6 contains some conclusions.

\section{From Global Efficiency to Net-Ability}

Global efficiency from the $\mathrm{CN}$ theory was initially defined by Latora $[2,4,10]$ and was later widely used to evaluate performance of network, such as vulnerability assessment or location of critical components $[4,26]$. It was also utilized in analyzing cascading failures for assessing power systems [2729]. The definition for global efficiency can be written as

$$
E(\mathbf{Y})=\frac{1}{N(N-1)} \sum_{i \neq j} \frac{1}{d_{i j}} .
$$

$d_{i j}$ is the distance (length of the shortest path) between the pair of node $i$ and node $j$, and $N$ is the number of all nodes in the network. This formula is aimed at measuring network efficiency of information transmission by considering that the efficiency for sending information between a pair of nodes $i$ and $j$ is proportional to the reciprocal of their geodesic distance. For obtaining the general efficiency of the whole network, performance between each pair of nodes should be assessed to calculate an average value.

However, when applying this metric to evaluate power grids, the original concept of distance in (1) is not meaningful in electrical engineering $[2,4,30]$. Distance in power grids should be adjusted as the ability to overcome difficulties of transferring power between the pair of generator node $g$ and load node $d$. Through considering the economic and technical aspects, power transmission difficulties should depend both on power flow capacity and on impedance. According to the electrical circuit theory, the equivalent impedance between bus $g$ and bus $d$ can be expressed as

$$
Z_{g}^{d}=\frac{U_{g}^{d}}{I_{g}}=z_{g g}-2 z_{g d}+z_{d d} .
$$

$z_{g g}, z_{g d}$, and $z_{d d}$ are corresponding elements of the impedance matrix of the network.

Furthermore, the maximum power flow limit from $g$ to $d$ should also be considered, which can be calculated as

$$
C_{g}^{d}=\min _{l \in L}\left(\frac{C_{l}^{\max }}{\left|f_{l}^{g d}\right|}\right) .
$$

$C_{g}^{d}$ is power transmission capacity between the generatorload $(g-d)$ pair. $L$ is the set of all lines connecting $g$ and $d ; C_{l}^{\max }$ is the maximum power flow capacity for line $l ; f_{l}^{g d}$ is the power transfer and distribution factor (PTDF) when transferring power from bus $g$ to $d$. PTDF here has overcome the assumption of transferring physical quantity only through the shortest path in global efficiency. Therefore, with the new definition of distance in power grids and taking into account power flow capacity characterized by PTDF, net-ability for power transmission network was defined as $[3,4,9]$

$$
A(\mathbf{Y})=\frac{1}{N_{G} N_{D}} \sum_{g \in G} \sum_{d \in D} \frac{C_{g}^{d}}{Z_{g}^{d}} .
$$


$G$ and $D$ are, respectively, the sets of generator buses and load buses. $N_{G}$ is the number of generator buses and $N_{D}$ is the number of loads buses.

With fast development of smart grid technologies, distribution networks with DGs and BESS have become a hot topic in research and engineering. However, up to now, pure structural analysis has seldom been applied to this field. In fact, the evaluation of power supply security with penetration of DGs and BESS is meaningful and necessary; and site allocation for DGs and BESS from structural perspective can also contribute to real system planning and operation.

Although net-ability has been applied to evaluate structure vulnerabilities of power transmission network as an effective approach, it is not reasonable to directly apply it for modern distribution networks.

Firstly, only the capacity of transmission channel between any pair of generator bus and load bus was considered, because net-ability only targeted features of network and did not consider generators or loads as a part of networks. However, the power capacities of power sources and loads may impact on the final power supply performance if they are much different from the capacity of channel, especially for distribution networks where the capacities of power sources are not comparable with transmission networks. Therefore, the connotation of structural analysis should not be limited to pure topological features but should also include some static physical features of devices, such as capacities of power sources and BESS.

Secondly, in definition of net-ability, all generator buses are supposed to have large capacity to support long distance transmission, so any generator can be a power source for any load bus in the same network. However, in distribution networks, most DGs have much smaller power capacity and may only be effective power sources for a range of local loads. Therefore, DGs cannot be considered as equal power sources in set $G$ with other traditional sources and cannot be an effective power source for any load bus in the network.

Thirdly, most DGs are renewable power generation, such as wind power or solar power. The output power of such power sources greatly depends on the availability of primary energy. Therefore, with intermittency and fluctuation, these power sources cannot supply continuous full rated output power for any required time period. Similar problem exists for BESS, which have to operate in charging and discharging modes in turn and cannot supply continuous full rated power for any required time period. As these features can seriously impact on the power supply performance of the distribution networks, they should not be neglected in structural analysis.

\section{Power-Supply-Ability for Distribution Networks}

To address the problems mentioned above, a new metric named as Power-Supply-Ability (PSA) dedicated to distribution networks is proposed based on net-ability.

To address the first problem mentioned in Section 2, the capacity of power source will be compared with the capacity of transmission channel defined in (3), and the minimum one will be applied in evaluating Power-Supply-Ability:

$$
\frac{\min \left(C_{g}^{d}, C_{g}\right)}{Z_{g}^{d}}
$$

If the capacity of power source is smaller than the channel capacity, that means the channel capacity cannot be fully utilized by the power source, so it cannot be directly used in evaluating Power-Supply-Ability.

As for the second problem in Section 2, with development of renewable technology, focus of environmental-friendly energy, and demand/supply balance, new generation technologies of DG such as wind generation and energy storage are gradually considered in distribution networks [17, 31, 32]. However, according to their common small capacities $[32,33]$ and power generating efficiency, they should not be directly evaluated as above conventional power sources. So in definition of PSA, DGs will only be considered as backup or auxiliary power sources to improve power supply reliability and quality, which cannot be considered in a source-load pair in average calculation, but just as an additional compensation for a conventional source-load pair. Therefore, the net-ability can be extended as

$$
\operatorname{PSA}(\mathbf{Y})=\frac{1}{N_{G} N_{D}}\left\{\sum_{g \in G}\left[\sum_{d \in D}\left(\frac{\min \left(C_{g}^{d}, C_{g}\right)}{Z_{g}^{d}}+\sum_{g_{B} \in G_{B}^{d}} \frac{\min \left(C_{g_{B}}^{d}, C_{g_{B}}\right)}{Z_{g_{B}}^{d}}\right)\right]\right\},
$$

where $G_{B}^{d}$ is the set of DG buses which are effective power sources for load bus $d$. And $C_{g_{B}}$ is capacity of DG at bus $g_{B} ; C_{g_{B}}^{d}$ is power transmission capacity from bus $g_{B}$ to load $d$, which can also be calculated by (3). $Z_{g_{B}}^{d}$ is the equivalent impedance between $g_{B}$ and the supplied load bus $d$. The metric of net-ability calculates average network performance for all possible (generation + load) pairs. PSA considers a power supply layout in terms of main power source + load + auxiliary sources and calculates average power supply performance for all possible power supply layouts. This means that when considering the power supply of one pair $(g-d)$, additional power supply from local power supplies (DGs) to that load $d$ will also be considered to improve the supply performance.

Since DGs in (6) are considered to have small capacities in this paper, it implies that DGs could not serve any load in the network like conventional power sources. It then needs a method to identify effective DG power sources $G_{B}^{d}$ for a load bus $d$. From another point of view, we can say that we need a method to identify effective supply area of a DG bus $g_{B}$. 


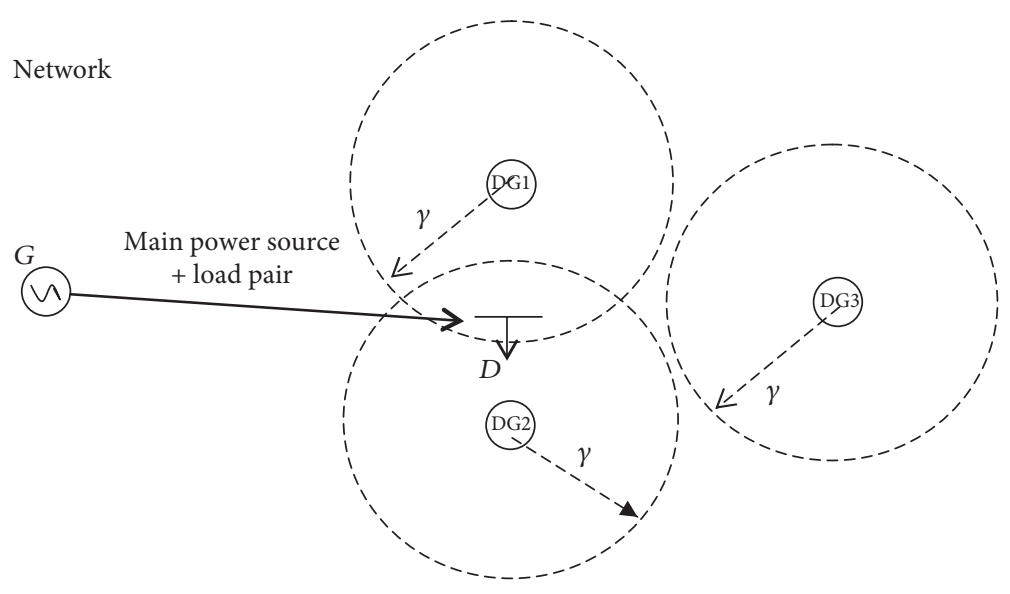

FIGURE 1: Sketch for effective supply area of DGs.

Then $G_{B}^{d}$ is the set of DG buses whose supply areas all include load $d$. When considering effective supply area of DGs, the general idea is that, with the same impedance, higher power supply capacity increases the efficient supplying area; with the same power supply capacity, higher impendence reduces the efficient supplying area. Then, a criterion to identify effective supply area for a DG bus is proposed as

$$
\frac{Z_{g_{B}}^{d}}{\min \left(C_{g_{B}}^{d}, C_{g_{B}}\right)} \leq \gamma, \quad g_{B} \neq d
$$

All DGs from $G_{B}^{d}$ satisfy criterion $Z_{g_{B}}^{d} / \min \left(C_{g_{B}}^{d}, C_{g_{B}}\right) \leq \gamma$ and $g_{B} \neq d$. To be specific, when the criterion is satisfied, it means that the DG has the ability to supply power to load $\mathrm{d}$ with acceptable power quality. $g_{B} \neq d$ means that when analyzing power supplied to load $d$, any DG directly installed at load bus $d$ will not be evaluated. The reason is that PSA in this paper is to evaluate the distribution network; once the DGs are placed at the load bus $d$, to some degree, they could not be considered as a part of network because their interactions are not through the network frame, and the power supply from these DGs to load $d$ should be considered as an internal supply, which is isolated from the whole system performance. Additionally, $\gamma$ is a parameter to define the DG supply area and it should be a value based on statistical analysis and simulation.

Figure 1 is a sketch to explain the meaning of effective supply area and power supply layout. A main power source $\mathrm{G}$ and a load bus D can be considered as a generation + load pair. There are three different DGs in the figure. Each DG could be considered as the center of a circle whose radius is $\gamma$. The circles could be considered as the effective area of each DG. The load bus D is inside the circles of DG1 and DG2 but outside of the circle of DG3. That means DG1 and DG2 are all effective auxiliary sources for $\mathrm{D}$ and should be included in $G_{B}^{d}$. And DG3 is not an effective auxiliary source for D; it should not be included in $G_{B}^{d}$. So the corresponding power supply layout could be $(\mathrm{G}+\mathrm{D}+\mathrm{DG} 1+\mathrm{DG} 2)$.

To address the third problem mentioned in Section 2, as some renewable DGs and BESS cannot supply continuous full rated power, a power supply factor is introduced:

$$
\eta=\frac{T_{S}}{T_{P}}=\frac{\text { Equivalent Supply Time at Rated Power }}{\text { Total Time of One period }}
$$

For a DG or BESS with rated power $P$, suppose, in one calculating period $T_{P}$, the accumulated output energy is $E_{P}$; then the equivalent supply time at rated power during this period can be calculated as

$$
T_{S}=\frac{E_{P}}{P}
$$

This is a method to unify all main power sources, DGs and BESS systems. For example, for a main power source $g$, such as a distribution substation which has ability to supply rated power during any required time period, the corresponding power supply factor $\eta_{g}$ is equal to 1 . This will not influence the calculation of net-ability. But for some DGs and BESS, this power supply factor needs to be calculated according to statistical data. For a wind power generator or PV panel, this needs to select a long term period with corresponding weather information and actual output energy statistical data. For BESS, the system needs specific time for charging and then to make discharging for some time. The power supply factor should be calculated based on an average charging and discharging period.

Furthermore, the power supply factor is an indicator to reflect the ability of a power source to make continuous rated power supply but not description for real operating state. For example, a main power source (distribution substation) may have power supply factor of 1 meaning ability to supply rated power for any required time period, but it does not mean it always operates at rated power for any time. 
With this power supply factor, PSA can be finally defined as

$$
\operatorname{PSA}(\mathbf{Y})=\frac{1}{N_{G} N_{D}}\left\{\sum_{g \in G}\left[\sum_{d \in D}\left(\eta_{g} \frac{\min \left(C_{g}^{d}, C_{g}\right)}{Z_{g}^{d}}+\sum_{g_{B} \in G_{B}^{d}} \eta_{g_{B}} \frac{\min \left(C_{g_{B}}^{d}, C_{g_{B}}\right)}{Z_{g_{B}}^{d}}\right)\right]\right\} \quad\left(g \neq d, g_{B} \neq d\right)
$$

Similar to calculation for net-ability, the relative change of PSA after removal of components can help to indicate the most important components in the targeted distribution network:

$$
\Delta \mathrm{PSA}=\frac{\operatorname{PSA}(Y)-\operatorname{PSA}(Y-1)}{\operatorname{PSA}(Y)},
$$

where $\operatorname{PSA}(Y-1)$ represents the Power-Supply-Ability of distribution network after one component is removed and $\triangle \mathrm{PSA}$ is the relative drop of PSA normalized by $\operatorname{PSA}(Y)$ to identify critical components of a network. Higher value of $\triangle$ PSA implies that this component is more critical and removal of it could result in severer negative effects. Thus, this component needs more protection.

\section{Overall Ability of Networks for DG Integration}

In the field of complex networks, it has been widely acknowledged that the performance and vulnerabilities of networked infrastructures are tightly related to the original topology and structure. Furthermore, in analyzing performance and security issues for power grids, topology and extended structural factors, such as positions of generation and load buses, capacities of transmission lines, and contributions of all paths in power transmission have been taken into account. However, up to now, few research works have considered DGs in terms of their positions and capacities as structural factors and evaluated their possible contributions in improving network performance and security. This is the original motivation to propose PSA.

However, it is important to point out that such improvements are from bidirectional interactions between the original networks and the integrated DGs. Therefore, the final effects may depend not only on the features of DGs, but also on some inherent features of the original networks. In these inherent features, topology and structural factors of the original networks are very important. Therefore, there should be two different perspectives to consider the issues about integration of DGs with distribution networks. The first perspective is at a local level and from individual viewpoint of DGs to discuss possible optimal deployment and operation of DGs with structural factors as given conditions. The second perspective should be at a global level and from overall viewpoint of original networks to discuss the inherent potential abilities of networks about how their performance and security can be improved by penetration of DGs. That is to say, with the same extents of DG penetration, the extents of improvement for different distribution networks may be different due to their inherent characteristics, such as topology and structure. Up to now, most existent works related to DGs were from the first perspective; few works were from the second perspective or even aware of its existence. In this paper, we will propose methodologies based on PSA to evaluate and compare this inherent potential of networks from the second perspective.

The inherent potential abilities of networks for improvement by DG penetration are related to two aspects, that is, network performance and security. The first issue is to discuss how the network static performance can be improved by same extent of DG penetration. The second issue is to discuss how the vulnerabilities of the networks can be reduced by penetration of DGs.

For the first issue, to make overall evaluation, two networks can be considered, the original network $Y$ and a modified network $Y+T$. In $Y+T$, we suppose all buses of $Y$ are connected to DGs with same power supply factor and capacity; then the variance of PSA before and after adding these DGs could be used to evaluate the original system structure for its inherent ability to be improved by DGs.

Alternatively, we may suppose a unique DG with given power supply factor and capacity is connected to each bus of $Y$ in turn; the increase of PSA corresponding to each bus could be used to evaluate which one may be the best location to install a DG. But, furthermore, we may also calculate the average value of increase in PSA for all buses to make overall evaluation for inherent ability of the network.

Both methods can be applied jointly to indicate the inherent ability of the network. The former one is mainly from an overall and accumulated point of view; the second one is mainly from an average point of view.

For the second issue, the vulnerability of the original network can be characterized by the relative drop of PSA calculated by (11); then the vulnerability of network $Y$ can be defined as

$$
\operatorname{VUL}(Y)=\max _{l \in L}\left\{\frac{\operatorname{PSA}(Y)-\operatorname{PSA}(Y-l)}{\operatorname{PSA}(Y)}\right\} .
$$

The most critical line $l_{m}$ can be identified as corresponding to the maximum PSA drop in (12). Similar to the former ideas, DGs with same power supply factor and capacity can be added to all buses of network $Y$, which can be indicated as $Y+T$. In $Y+T$, we can also calculate its maximum drop of PSA by (12). So this variance of PSA drop can reflect the original overall ability of the network to be improved for its security by adding DGs.

To give more detailed information, the adding of DGs can be performed according to the importance sequence of 


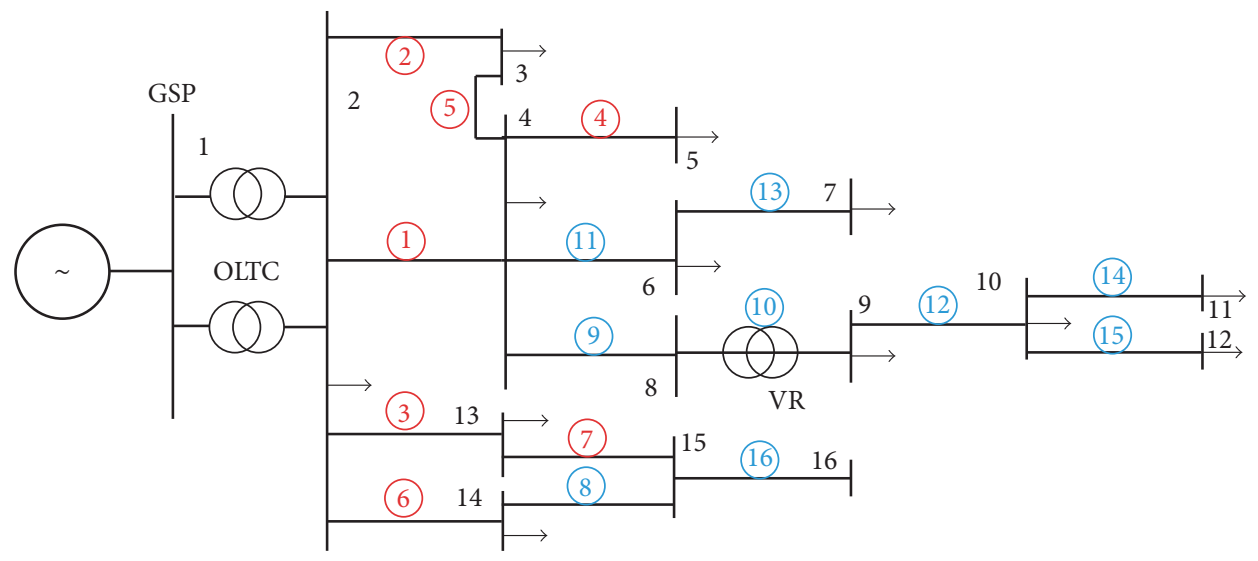

FIGURE 2: Ranking of branch in UKGDS_86.

buses step by step. The importance sequence is the sequence from larger to smaller PSA for different $\left(Y+D G_{i}\right)$. This may give direct expression of impacts from DGs on security for different locations.

For given networks, these methods can jointly evaluate and discover their difference in potential abilities to be improved by DG penetration. This can help analysis and decisions in overall planning and investment related to DG penetration. Possible application of these methods is that when limited resources of DG are given, if one distribution network needs to be selected from two or several candidate networks to install these DG resources, these methods can help to determine which one is the best choice according to their original structure features. And, furthermore, this also can help to identify structural bottlenecks of distribution networks in DG development.

\section{Case Study}

In this section, the PSA is applied to the IEEE-33-bus system and a real distribution network UKGDS_86. UKGDS_86 initially is tested without DGs and it will be assessed to compare and analyze corresponding power supply performance through PSA. The initial PSA for UKGDS_86 without DGs is calculated as

$$
\text { PSA }(\text { UKGDS_86) }=57.1253 \text {. }
$$

When removing one branch from UKGDS_86, performance of power supply could be evaluated through PSA $(Y-$ 1) metric and relative drop $\triangle P S A$ could be used to rank the critical lines.

As in Table 1, once a branch is removed, it could result in decreases in PSA. For better visual effect, rankings are shown in Figure 2.

The red numbers in red circles represent five branches which would result in significant PSA drops once removed and need more protection. As is shown, these branches mainly occur close to power station. It seems that branches near power station play significant roles in power supply; removal of them would influence performance of the whole
TABLE 1: Critical branches rankings in UKGDS_86.



system more severely and need more protection for grid security. Branches in blue, which are relevantly away from power station and near loads, will cause less negative consequences when they are removed.

When adding same DGs to each bus, which means there are now totally 16 DGs in network, PSA value of this new grid increases to 62.721. Similar to above analysis, branches of this grid are ranked and labeled as in Figure 3.

As is shown in Figure 3 comparing with former conventional network without DGs, removal of branches results in similar rankings. Only branch 2-3 and branch 4-5 have exchanged ranks, which are labeled in Figure 3, but they still keep top five as former. So installation of DGs can improve power supply performance of the network but does not change the protection properties of branches too much. 


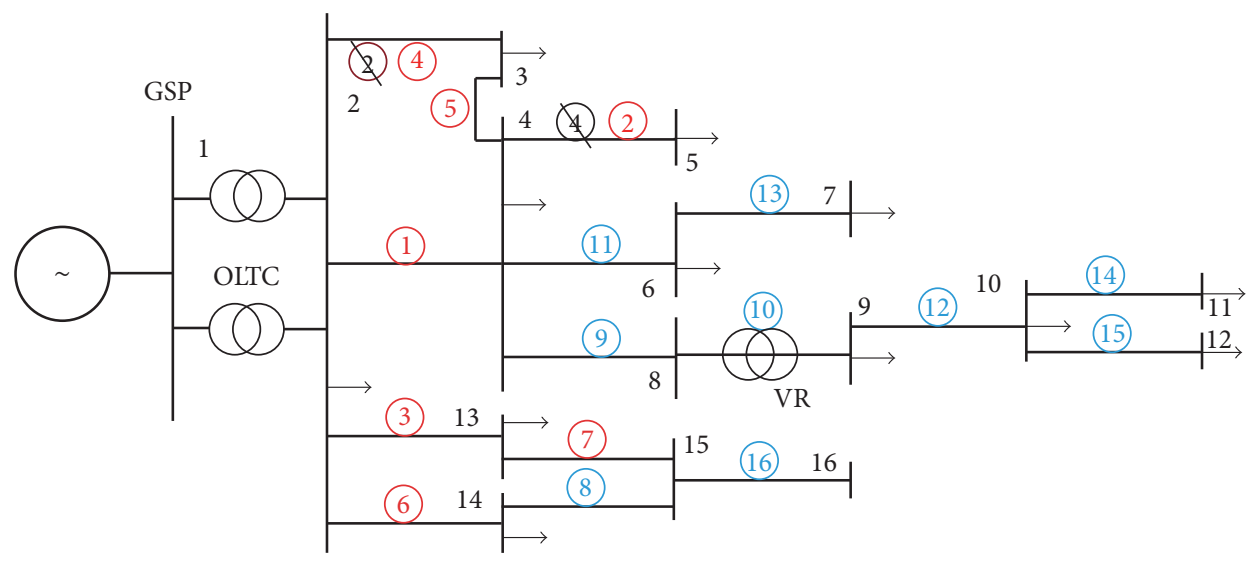

FIGURE 3: Ranking of branch in UKGDS_86 with DGs.

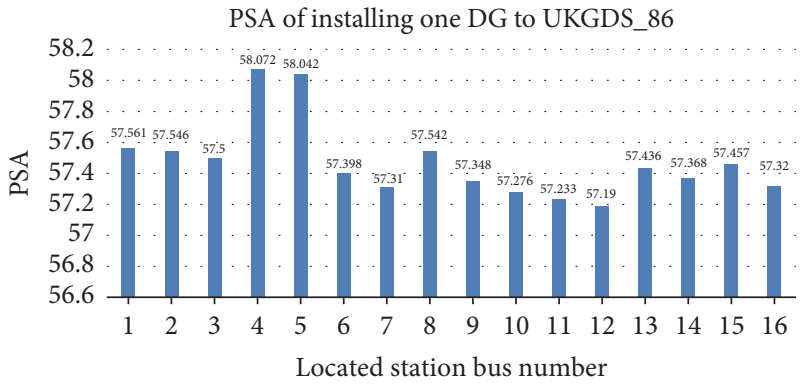

FIGURE 4: PSAs for adding one DG at different buses.

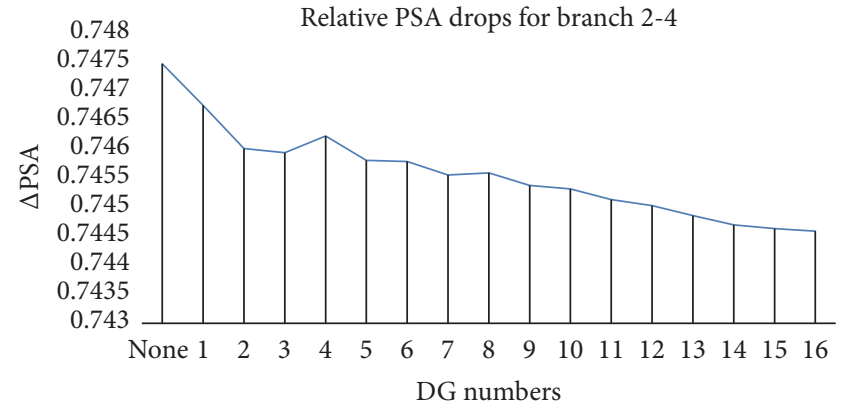

Figure 5: $\triangle$ PSA of branch 2-4 with different DGs.
Branches near power distribution station still need more attention for security.

To quantitatively identify which buses are best sites for installation of DG, one DG can be installed on each bus in turn and the corresponding increase of PSA is shown in Figure 4.

It shows that bus 4 is the best location for locating DG and bus 12 is the relevantly worst. Based on this, the first DG should be suggested to be located on bus 4 and then the second one will be added at bus 5 . Through considering overall performance, bus 12 with worst PSA $=57.19$ will be lastly planned to add a DG.

Based on the order in Figure 4, DGs are added one by one for further observation of DG influence. As is shown, initially there is no DG; based on orders in Figure 3, the first DG will be added to bus 4 and then add the second DG at bus 5 . Add DGs one by one under above suggestion order and record relative PSA drops ( $\triangle \mathrm{PSA}$ ) of (12) during DG addition procedure.

From Figures 2 and 3, branch 2-4 is the most critical line and needs the most protection. Thus, this branch is selected in Figure 5 to observe $\triangle$ PSAs under different number of DGs.

Figure 5 shows that, with increases of DGs, they generally decrease $\triangle P S A$; in other words, addition of local power supplies could improve power grids performance and decrease the vulnerability of critical lines. At the beginning, the curve is steeper and indicates that the adding of DGs at beginning could obviously reduce system vulnerabilities. However, at the end of the curve, it becomes flat and indicates that the effect of adding DGs becomes weak step by step.

Furthermore, an ideal small-scale generation with power supply factor of 1 is utilized to test IEEE-33-bus system and compare with analytical methods reported earlier [34] through considering improvement of voltage profile and reduction of losses.

In Figure 6, the $y$-axis of ranking represents the performance of DG installation, the minimum number means best performance, and the largest number means the worst performance. For instance, in purple line, when DG location is 5 , its priority ranking is 1 and it means that installing DG to bus location 5 will improve performance most.

There are three lines in Figure 6, the blue line is the ranking results from PSA metric in this paper, which is based on structural analysis. While voltage stability index and Exhaustive Load Flow Method (ELF) [34-36] are based on detailed power flow calculations, involving detailed load voltages, generator voltages, and load currents. These methods consider that addition of DGs may increase real power flow back to system and then cause voltage rise or may increase reactive power follow into feeder and then cause voltage to fall [34-36]. Thus, reduction of losses, which is aimed at minimizing total power losses and improvement of voltage stability, is the main purpose for these methods to consider DG optimum location. To some extent, methods 


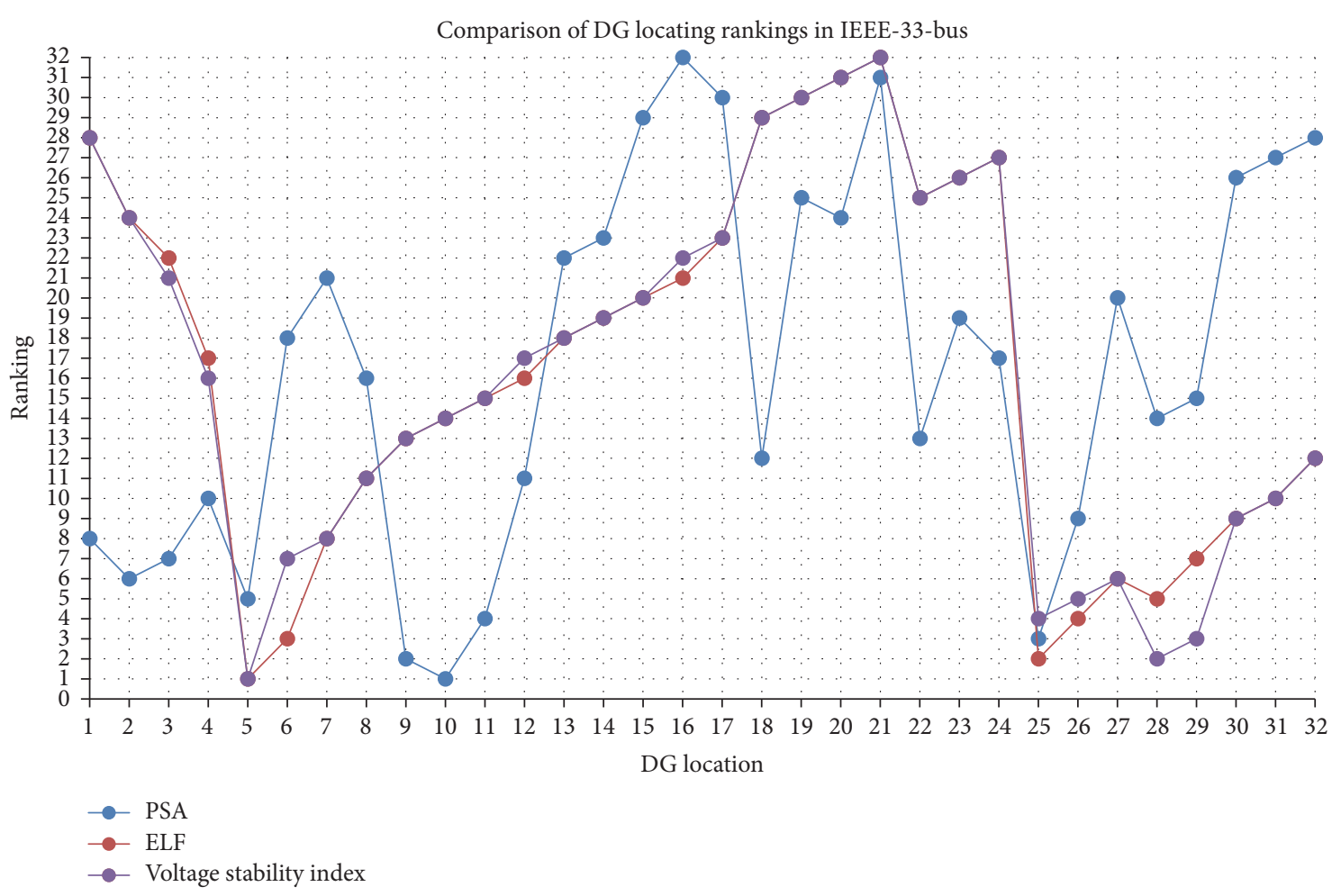

FIGURE 6: PSAs for installing DGs at different buses.

based on states pay more attention to economy and quality while PSA method based on structural analysis focuses on overall performance and security.

In other words, analyses based on operating states could not consider all possible system operative states through calculating and they mostly focus on economy through limited situations. However, security analysis should pay attention to emergencies such as intentional attacks, which are not the common conditions. Thus, safety analysis and above economic analysis have contradictions.

Since analyzing aspects of three methods are different, there are variances of rankings in Figure 6. However, there are still some installation locations with relevantly smaller priority variances. For these locations, they contain both aspects of security and aspects of economy and quality, which are more meaningful.

In Figure 7, bus locations 25, 5, and 26 have high rankings from perspectives of economy, quality, and security, which should be firstly suggested to install DGs while bus locations 13,14 , and 21 have low performance from three perspectives and may not be recommended to add DGs. There are also inconsistent locations such as bus locations 6, 9, 10, and 28 . That means evaluation by limited operational states cannot identify possible problems in security analysis which is the contribution of the measure from this paper.

\section{Conclusions}

Structural analysis based on complex network theory has been considered promising for analyzing security issues

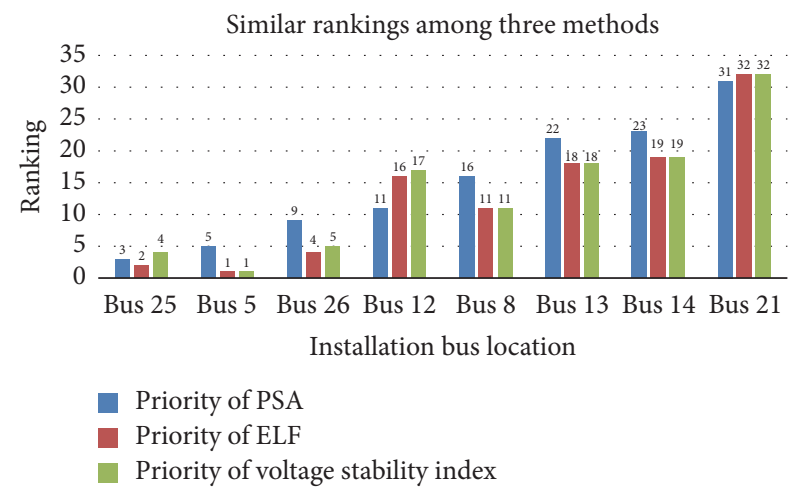

FIGURE 7: Similar rankings among three methods.

of power grids. However, the meaning of "structure" has normally been limited to topological connections or some related physical features of networks. In fact, in broad sense, some static physical features of related components may also have tight relation with network performance, such as capacities, effective supply area, or intermittency of generators. Many effective metrics of complex networks are based on statistic evaluation. And these static physical features can also provide great valuable information from statistic perspective. Therefore, it is inevitable that these more static features would be integrated with network structures when the targeted issues are more specific and professional.

At the same time, with fast development of smart grid technology, modern power distribution networks with more 
DGs and BESS have taken on more challenges in operation and security issues. By consideration of some specific defects of former metrics for distribution networks and DGs, it is necessary to update related methodologies by some further important and special features to extend the meaning of structural analysis. This paper proposed a dedicated metric named as Power-Supply-Ability for power distribution networks based on net-ability which was formerly applied to power transmission networks. Three defects of net-ability applying to distribution networks with DGs are identified. Based on solutions to these defects, the net-ability is updated and refined as Power-Supply-Ability for distribution networks. Furthermore, it is pointed out that it is an inherent ability depending on the original network structure to improve its performance and security by same extent of DG penetration. Through case studies, this method has been proved to be effective in identifying potential structural vulnerabilities of distribution networks. Furthermore, it can help in site allocation for DGs and complementing other methods which are only based on limited analysis of states. The analysis and judgment for DG plan and operation should consider perspectives of economy, quality, and security. This metric can help to construct a more comprehensive methodology. In the future, this method will try to be applied to real world systems in decision of DG allocation for further development and analysis.

\section{Conflicts of Interest}

The authors declare that there are no conflicts of interest regarding the publication of this paper.

\section{Acknowledgments}

This work has been supported by the SDIC Baiyin Wind Power Co., Ltd., through the joint research project "Study on Key Technologies for Energy-Internet-Oriented Microgrids.”

\section{References}

[1] C. Liu, Q. Xu, Z. Chen, and C. L. Bak, "Vulnerability evaluation of power system integrated with large-scale distributed generation based on complex network theory," in Proceedings of the 47th International Universities Power Engineering Conference (UPEC '12), 2012.

[2] E. Bompard, D. Wu, and F. Xue, "Structural vulnerability of power systems: A topological approach," Electric Power Systems Research, vol. 81, no. 7, pp. 1334-1340, 2011.

[3] E. Bompard, R. Napoli, and F. Xue, "Analysis of structural vulnerabilities in power transmission grids," International Journal of Critical Infrastructure Protection, vol. 2, no. 1-2, pp. 5-12, 2009.

[4] S. Arianos, E. Bompard, A. Carbone, and F. Xue, "Power grid vulnerability: a complex network approach," Chaos: An Interdisciplinary Journal of Nonlinear Science, vol. 19, no. 1, Article ID 013119, 2009.

[5] V. Latora and M. Marchiori, "Vulnerability and protection of infrastructure networks," Physical Review E: Statistical, Nonlinear, and Soft Matter Physics, vol. 71, no. 1, Article ID 015103, 2005.
[6] R. Albert, I. Albert, and G. L. Nakarado, "Structural vulnerability of the North American power grid," Physical Review E: Statistical, Nonlinear, and Soft Matter Physics, vol. 69, no. 2, Article ID 025103, 2004.

[7] D. J. Watts and S. H. Strogatz, "Collective dynamics of 'smallworld' networks," Nature, vol. 393, no. 6684, pp. 440-442, 1998.

[8] A. L. Barabasi and R. Albert, "Emergence of scaling in random networks," American Association for the Advancement of Science: Science, vol. 286, pp. 509-512, 1999.

[9] E. Bompard, R. Napoli, and F. Xue, "Extended topological approach for the assessment of structural vulnerability in transmission networks," IET Generation, Transmission \& Distribution, vol. 4, no. 6, pp. 716-724, 2010.

[10] L. D. F. Costa, F. A. Rodrigues, G. Travieso, and P. R. V. Boas, "Characterization of complex networks: a survey of measurements," Advances in Physics, vol. 56, no. 1, pp. 167-242, 2007.

[11] P. Hines, E. Cotilla-Sanchez, and S. Blumsack, "Do topological models provide good information about electricity infrastructure vulnerability?" Chaos: An Interdisciplinary Journal of Nonlinear Science, vol. 20, no. 3, Article ID 033122, 2010.

[12] U. Brandes and D. Fleischer, Centrality Measures Based on Current Flow, Springer, 2005.

[13] G. Chen, Z. Y. Dong, D. J. Hill, and G. H. Zhang, "An improved model for structural vulnerability analysis of power networks," Physica A: Statistical Mechanics and its Applications, vol. 388, no. 19, pp. 4259-4266, 2009.

[14] E. Bompard, D. Wu, and F. Xue, "The concept of betweenness in the analysis of power grid vulnerability," in Proceedings of the Complexity in Engineering (COMPENG '10), pp. 52-54.

[15] S. Arianos, E. Bompard, A. Carbone, and F. Xue, "Power grid vulnerability: A complex network approach," Chaos: An Interdisciplinary Journal of Nonlinear Science, vol. 19, no. 1, Article ID 013119, 2009.

[16] A. Oudalov, D. Chartouni, and C. Ohler, "Optimizing a battery energy storage system for primary frequency control," IEEE Transactions on Power Systems, vol. 22, no. 3, pp. 1259-1266, 2007.

[17] Y. Hida, Y. Ito, R. Yokoyama, and K. Iba, "A study of optimal capacity of PV and battery energy storage system distributed in demand side," in Universities Power Engineering Conference (UPEC), pp. 1-5, 2010.

[18] W. Y. Yang, X. Y. Yang, and J. J. Yang, "Research on distributed generation and application in power system," Advances of Power System \&amp; Hydroelectric Engineering, vol. 24, pp. 39-43, 2008.

[19] K. J. Qian and Y. Yuan, "Distributed generation technology and its impact on power systems," Relay, vol. 35, pp. 25-29, 2007.

[20] U. Doe, The potential benefits of distributed generation and rate-related issues that may impede their expansion, 2007.

[21] A. Yadav and L. Srivastava, "Optimal placement of distributed generation: an overview and key issues," in Proceedings of the International Conference on Power Signals Control and Computations (EPSCICON '14), 2014.

[22] A. Borbely and J. F. Kreider, Distributed generation: the power paradigm for the new millennium, CRC press, 2001.

[23] M. K. Maribu, Distributed Generation in Liberalised Electricity Markets [Ph.D. thesis], 2002.

[24] Y. Y. Wang, S. W. Mei, Y. B. Mao, and F. Liu, "Vulnerability assessment of power grid with distributed generation based on complex network theory," Journal of Systems Science and Mathematical Sciences, vol. 30, no. 6, pp. 859-868, 2010. 
[25] F. Gullí, "Small distributed generation versus centralised supply: a social cost-benefit analysis in the residential and service sectors," Energy Policy, vol. 34, no. 7, pp. 804-832, 2006.

[26] P. Crucitti, V. Latora, and M. Marchiori, "Locating critical lines in high-voltage electrical power grids," Fluctuation and Noise Letters, vol. 5, no. 2, pp. L201-L208, 2005.

[27] R. Kinney, P. Crucitti, R. Albert, and V. Latora, "Modeling cascading failures in the North American power grid," The European Physical Journal B, vol. 46, no. 1, pp. 101-107, 2005.

[28] B. C. Lesieutre, S. Roy, V. Donde, and A. Pinar, Power system extreme event screening using graph partitioning, Lawrence Berkeley National Laboratory, 2006.

[29] J. Li, "Identification of Cascaded Generator Over-Excitation Tripping Events," ProQuest, 2007.

[30] E. Bompard, E. Pons, and D. Wu, "Extended topological metrics for the analysis of power grid vulnerability," IEEE Systems Journal, vol. 6, no. 3, pp. 481-487, 2012.

[31] L. Kang, H. Guo, J. Wu, and S. Chen, "Characteristics of distributed generation system and related research issues caused by connecting it to power system," Power System Technology, 2010.

[32] R. Viral and D. K. Khatod, "Optimal planning of distributed generation systems in distribution system: a review," Renewable \& Sustainable Energy Reviews, vol. 16, no. 7, pp. 5146-5165, 2012.

[33] L. Li, "A study on distributed generation and its effects in power system," Journal of Modern Power Systems and Clean Energy, pp. 55-59, 2010.

[34] S. Kalambe, G. Agnihotri, and A. K. Bohre, "An Analytical Approach for Multiple DG Allocation," in Proceedings of the in Distribution System, vol. 2, 2013.

[35] D. Q. Hung and N. Mithulananthan, "Multiple distributed generator placement in primary distribution networks for loss reduction," IEEE Transactions on Industrial Electronics, vol. 60, no. 4, pp. 1700-1708, 2013.

[36] N. Acharya, P. Mahat, and N. Mithulananthan, "An analytical approach for DG allocation in primary distribution network," International Journal of Electrical Power \& Energy Systems, vol. 28, no. 10, pp. 669-678, 2006. 


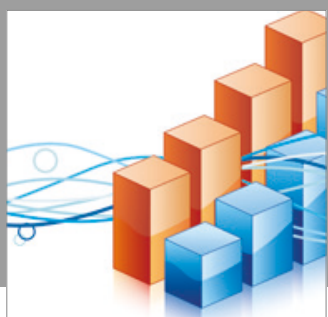

Advances in

Operations Research

vatersals

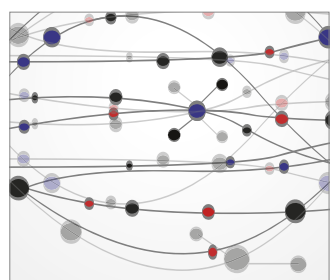

\section{The Scientific} World Journal
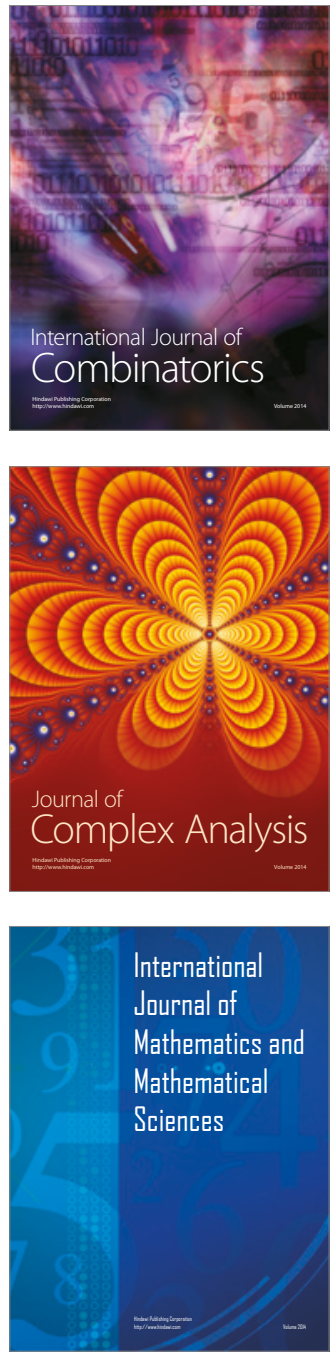
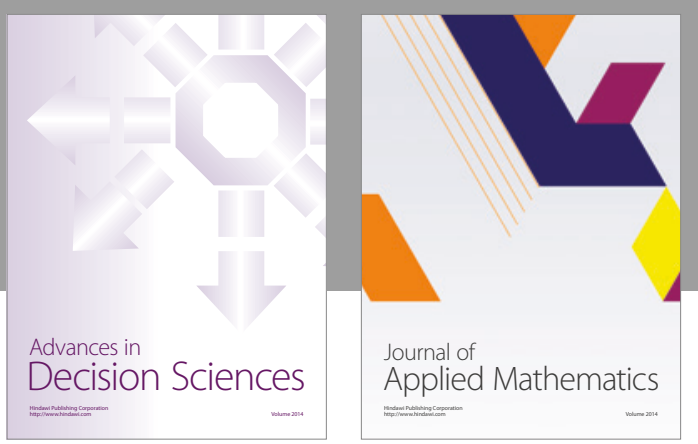

Algebra

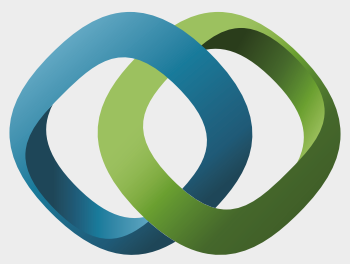

\section{Hindawi}

Submit your manuscripts at

https://www.hindawi.com
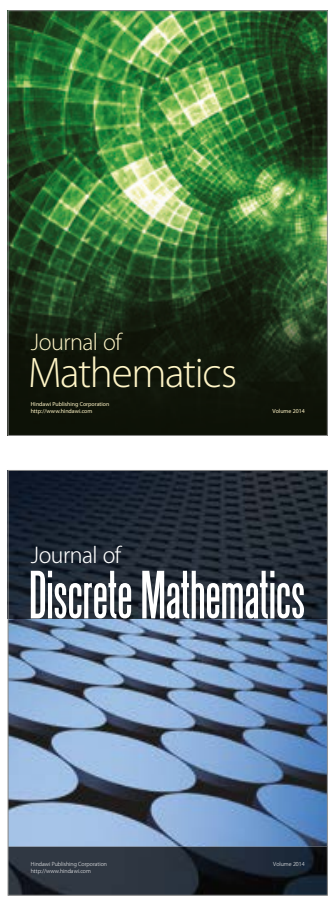

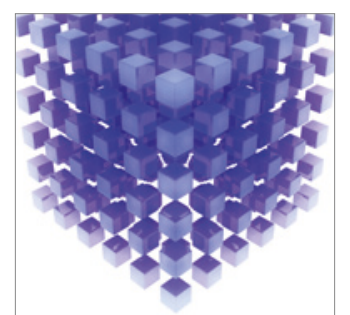

Mathematical Problems in Engineering


Journal of

Function Spaces

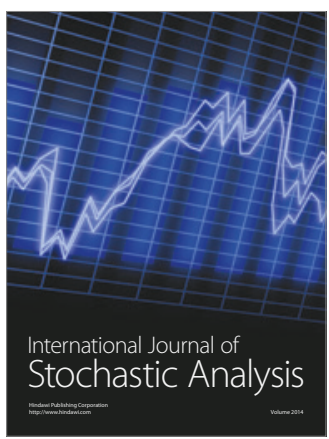

Probability and Statistics
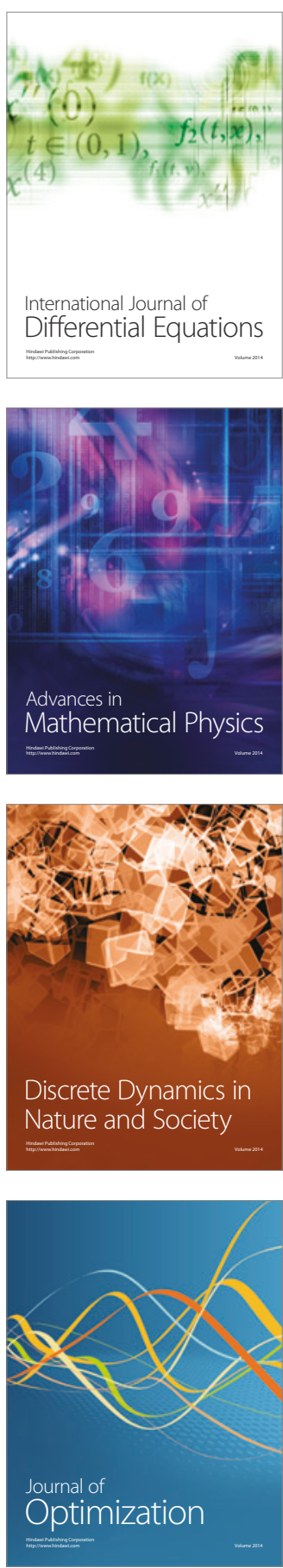